\title{
SINR Distribution for LTE Downlink Multiuser MIMO Systems
}

\author{
Zihuai $\operatorname{Lin}^{\dagger}$, Pei Xiao ${ }^{\dagger \dagger}$, Branka Vucetic ${ }^{\dagger}$ \\ †University of Sydney, 2006 Sydney, Australia \\ ${ }^{\dagger \dagger}$ Queen's University Belfast, BT3 9DT, United Kingdom
}

\begin{abstract}
The LTE downlink multiuser Multiple Input Multiple Output (MIMO) systems are analyzed in this paper. Two Spatial Division Multiplexing (SDM) multiuser MIMO schemes are investigated: Single User (SU) and Multi-user (MU) MIMO schemes. The main contribution of this paper is the establishment of a mathematical model for the Signal to Interference plus Noise Ratio (SINR) distribution for multiuser SDM MIMO systems with frequency domain packet scheduler.
\end{abstract}

Keywords: LTE, OFDMA, Multiuser MIMO, SINR.

\section{INTRODUCTION}

In 3GPP Long Term Evolution (LTE) (also known as Evolved-UMTS Terrestrial Radio Access (E-UTRA)), Multiple-Input Multiple-Output (MIMO) and Orthogonal Frequency Division Multiple Access (OFDMA) have been selected for downlink transmission [1]. Both Spatial Division Multiplexing (SDM) and Frequency Domain Packet Scheduling (FDPS) have been proposed. SDM is used to improve the spectral efficiency of the system, while FDPS allows the packet scheduler at the Base Station (BS) to exploit the available multiuser diversity in both time and frequency domain. In [2], it is shown that the MIMO schemes with combined SDM and FDPS can further enhance the system performance.

Both open loop and closed loop $\mathrm{MIMO}^{1}$ are considered as possible solutions in 3GPP LTE. However, the use of closed loop provides both diversity and array gains, and hence a superior performance. Due to its simplicity and robust performance, the use of linear precoding has been widely studied as a closed loop scheme [2]. Here, we refer to the open loop MIMO as the SDM MIMO without precoding, and the closed loop MIMO as the linearly precoded SDM MIMO. To the authors knowledge, theoretical analysis of linearly precoded multiuser SDM MIMO systems combined with FDPS has not been studied so far. In this paper, we conduct a theoretical analysis for SINR distribution in multiuser MIMO systems with SDMFDPS. Although our study is conducted for the 3GPP LTE downlink packet data transmission [1], the analysis method is generally applicable to other packet switched systems.

\section{SySTEM MODEL}

In this section, we describe the system model of multiuser SDM MIMO schemes for 3GPP LTE downlink transmission with packet scheduling. The basic scheduling unit in LTE is the Physical Resource Block (PRB), which consists of a number of consecutive OFDM sub-carriers reserved during the transmission of a fixed number of OFDM symbols. One PRB of 12 contiguous subcarriers can be configured for localized transmission $^{2}$ in a sub-frame. With the localized transmission

\footnotetext{
${ }^{1}$ Open loop and closed loop MIMO correspond to the MIMO systems without and with channel state information at the transmitter, respectively [1].

${ }^{2}$ In the localized FDMA transmission scheme, each user's data is transmitted by consecutive subcarriers, while for the distributed FDMA transmission scheme, the user's data is transmitted by distributed subcarriers [1].
}

scheme, two SDM schemes are now under investigation [1], i.e., Single User (SU) MIMO and Multi-User (MU) MIMO schemes. They differ in terms of the freedom allowed to the scheduler in the spatial domain [1]. With SU-MIMO scheme, only one single user can be scheduled per PRB; whereas with MU-MIMO scheme, multiple users can be scheduled per PRB, one user for each sub-stream per PRB.

The Frequency Domain (FD) scheduling algorithm considered in this work is the FD Proportional Fair (PF) [3] packet scheduling algorithm, which is being investigated under LTE. It is shown in [4] that a scheduler is PF if the instantaneous rate $\left\{R_{k, i}\right\}$ maximizes $\left[\frac{R_{k, i}}{\overline{R_{k, i}}}\right]$, where $\overline{R_{k, i}}=$ $\left(1-\frac{1}{T_{w i n}}\right) \overline{R_{k-1, i}}+\frac{1}{T_{w i n}} R_{k, i}$ is the moving average of the maximum achievable rate of user $i$ at the $k$ th time slot over a sliding window of $T_{\text {win }}$ time slots. With simplifying assumptions similar to [5], i.e., user's fading statistics are independent and identically distributed, $T_{w i n}$ is sufficiently large so that the average received user data rates are stationary, and there is approximately a linear relationship between the throughput and the SINR. The PF scheduling algorithm can be regarded as a selection diversity process, which selects the users with the best unified effective SINR. The unified effective SINR is defined as the equivalent single stream SINR which offers the same instantaneous (Shannon) capacity as a MIMO scheme with multiple streams [6]. Let $\gamma_{i}, i \in\{1, \cdots, k\}$ be the SINR of each sub-stream, and $\gamma_{u}$ be the unified effective SINR, then $\log _{2}\left(1+\gamma_{u}\right)=\sum_{i} \log _{2}\left(1+\gamma_{i}\right)$, therefore, $\gamma_{u}=\prod_{i}\left(1+\gamma_{i}\right)-1$. The distribution of $\gamma_{u}$ can be derived given the distribution of $\gamma_{i}$. The purpose of introducing unified SINR is to facilitate the SINR comparison between SU MIMO and MU MIMO schemes.

The system considered here has $n_{t}$ transmit antennas at the Base Station (BS) and $n_{r}^{i}$ receive antennas at the $i$ th Mobile Station (MS), $i=1,2, \cdots, K$. Without loss of generality, we assume that all the MSs have equal numbers of antennas $n_{r}$, and define $M=\min \left(n_{t}, n_{r}\right)$ and $N=\max \left(n_{t}, n_{r}\right)$. The number of users simultaneously served on each PRB for the MU-MIMO scheme is usually limited by the number of transmitter antennas $n_{t}$. The scheduler in BS select at most $n_{t}$ users per PRB from the $K$ active users in the cell for data transmission. Denote by $\zeta_{k}$ the set of users scheduled on the $k_{t h}$ PRB and $\left|\zeta_{\mathbf{k}}\right|=n_{t}$. The received signal vector at the $j$ th MS, $j \in \zeta_{n}$, can be modeled as $\mathbf{y}_{n, j}=\mathbf{H}_{n, j} \mathbf{x}_{n}+\mathbf{n}_{n, j}$, where $\mathbf{n}_{n, j} \in \mathbb{C}^{n_{r} \times 1}$ is a circularly symmetric complex Gaussian noise vector with a zero mean and covariance matrix $N_{0} \mathbf{I} \in \mathbb{R}^{n_{r} \times n_{r}}$, i.e., $\mathbf{n}_{n, j} \sim \mathcal{C N}\left(\mathbf{0}, N_{0} \mathbf{I}\right) ; \mathbf{H}_{n, j} \in \mathbb{C}^{n_{r} \times n_{t}}$ is the channel matrix between the BS and the $j$ th MS at the $n$th PRB and $\mathbf{x}_{n} \in \mathbb{C}^{n_{t} \times 1}$ is the transmitted signal vector at the $n$th PRB, and the $\mu$ th element of $\mathbf{x}_{n}$ is the data symbol $x_{n, \mu}$ transmitted from the $\mu$ th MS, $\mu \in \zeta_{n}$.

For a linearly precoded MIMO system, the received signal 
vector at the $j$ th MS, $j \in \zeta_{n}$, can be obtained by

$\mathbf{y}_{n, j}=\mathbf{H}_{n, j} \sum_{\mu \in \zeta_{n}} \mathbf{b}_{n, \mu} x_{n, \mu}+\mathbf{n}_{n, j}=\mathbf{H}_{n, j} \sum_{\mu \in \zeta_{n}} \mathbf{B}_{n, \mu} \boldsymbol{\psi}_{n, \mu}+\mathbf{n}_{n, j}$

where $\mathbf{b}_{n, \mu} \in \mathbb{C}^{n_{t} \times 1}$ is the beamvector for the $\mu$ th MS user data on the $n$th PRB and $\mathbf{B}_{n, \mu} \in \mathbb{C}^{n_{t} \times n_{t}}$ is the precoding matrix with the $\mu$ th column of $\mathbf{B}_{n, \mu}$ equal to $\mathbf{b}_{n, \mu}, \boldsymbol{\psi}_{n, \mu} \in$ $\mathbb{C}^{n_{t} \times 1}$ is a column vector in which the $\mu$ th element equal to $x_{n, \mu}$ and the rest equal to zero.

For MU-MIMO SDM scheme, we assume that the precoding matrices for scheduled users are approximately identical so that the same precoding matrix can be used for the scheduled users. Then (1) becomes

$$
\mathbf{y}_{n, j}=\mathbf{H}_{n, j} \mathbf{B}_{n, j} \sum_{\mu \in \zeta_{n}} \boldsymbol{\psi}_{n, \mu}+\mathbf{n}_{n, j}=\mathbf{H}_{n, j} \mathbf{B}_{n, j} \mathbf{x}_{n}+\mathbf{n}_{n, j}
$$

where $\mathbf{B}_{n, j}$ is the precoding matrix for the $j$ th MS on the $n$th $\mathrm{PRB}, j \in \zeta_{n}$.

With a linear Minimum Mean Square Error (MMSE) receiver, also known as a Wiener filter, the optimum precoding matrix under the sum power constraint can be generally expressed as $\mathbf{B}_{n, j}=\mathbf{U}_{n, j} \sqrt{\boldsymbol{\Sigma}_{n, j}} \mathbf{V}_{n, j}$ [8]. Here $\mathbf{U}_{n, j}$ is an $n_{t} \times n_{t}$ eigenvector matrix with columns corresponding to the $n_{t}$ largest eigenvalues of the matrix $\mathbf{H}_{n, j} \mathbf{H}_{n, j}^{H}$, where $\mathbf{H}_{n, j}^{H}$ is the Hermitian transpose of the channel matrix $\mathbf{H}_{n, j}$. For SchurConcave objective functions, $\mathbf{V}_{n, j} \in \mathbb{C}^{n_{t} \times n_{t}}$ is an unitary matrix, and $\boldsymbol{\Sigma}_{n, j}$ is a diagonal matrix with the $\eta$ th diagonal entry $\boldsymbol{\Sigma}_{n, j}(\eta, \eta)$ representing the power allocated to the $\eta$ th established data sub-stream, $\eta \in\left\{1,2, \cdots, n_{t}\right\}$.

\section{SINR DISTRIBUTION FOR OPEN LOOP SDM MIMO}

In the single user case with a $2 \times 2$ antenna MIMO scheme, with two data streams, uncorrelated Rayleigh fading channel, and a Zero Forcing (ZF) receiver, the Cumulative Distribution Function (CDF) of the unified effective SINR can be represented by [6]

$$
F_{\Gamma_{u}}^{s}(\gamma)=P_{r}\left(\Gamma_{u} \leq \gamma\right)=\int_{0}^{\gamma} \frac{2}{\gamma_{0}} e^{-\frac{2 x}{\gamma_{0}}}\left(1-e^{-\frac{2(\gamma-x)}{\gamma_{0}(1+x)}}\right) d x
$$

where $\Gamma_{u}$ is the unified effective SINR and $\gamma_{0}=E_{s} /\left(n_{t} N_{0}\right)$ where $E_{s}$ is the total transmit energy and $N_{0}$ is the power spectral density of the additive white Gaussian noise.

For localized downlink transmission with SU-MIMO SDM scheme and FD PF algorithm under the assumption of identical distribution of all effective SINR for all users, the probability that the SINR of a scheduled user is less than a certain threshold, i.e., the CDF of the post scheduling SINR per PRB can be computed as

$$
F_{\Gamma_{u}}^{O S}(\gamma)=\left[P_{r}\left(\Gamma_{u} \leq \gamma\right)\right]^{K}=\left[F_{\Gamma_{u}}^{s}(\gamma)\right]^{K},
$$

where $K$ is the number of active users in the cell or the so called User Diversity Order (UDO). That is the distribution of the best user, i.e., the largest SINR selected from the $K$ users.

Differentiating $F_{\Gamma_{u}}^{O S}(\gamma)$ yields the Probability Density Function (PDF) of the post scheduling SINR

$$
\begin{gathered}
f_{\Gamma_{u}}^{O S}(\gamma)=K\left[\int_{0}^{\gamma} \frac{2}{\gamma_{0}} e^{-\frac{2 x}{\gamma_{0}}}\left(1-e^{-\frac{2(\gamma-x)}{\gamma_{0}(1+x)}}\right) d x\right]^{K-1} \\
\cdot \int_{0}^{\gamma}\left[\frac{4}{\gamma_{0}^{2}(1+x)} \exp \left(-\frac{2\left(\gamma+x^{2}\right)}{\gamma_{0}(1+x)}\right)\right] d x .
\end{gathered}
$$

For an MU-MIMO SDM scheme, multiuser diversity can also be exploited in the spatial domain, which effectively increases the UDO. This is due to the fact that for localized transmission under an MU-MIMO scheme in LTE, we can schedule multiple users per PRB, i.e. one user per sub-stream. For a dual stream MU-MIMO scheme, the maximum number of users per PRB is 2 .

It was shown in [9] that for a spatial multiplexing MIMO scheme with $n_{t}$ transmit antennas and $n_{r}$ receive antennas, with a ZF receiver, the SINR on the $k$ th sub-stream has a Chi-squared PDF

$$
f_{\Gamma_{k}}^{s}(\gamma)=\frac{n_{t} \sigma_{k}^{2} e^{-n_{t} \gamma \sigma_{k}^{2} / \gamma_{0}}}{\gamma_{0}\left(n_{r}-n_{t}\right) !}\left(\frac{n_{t} \gamma \sigma_{k}^{2}}{\gamma_{0}}\right)^{\left(n_{r}-n_{t}\right)},
$$

where $\Gamma_{k}$ represents the instantaneous SINR on the $k$ th spatial sub-stream, $\sigma_{k}^{2}$ is the $k$ th diagonal entry of $\mathbf{R}_{\mathbf{t}}{ }^{-1}$ where $\mathbf{R}_{\mathbf{t}}$ is the transmit covariance matrix. Equ. (6) is for the flat Rayleigh fading channel with uncorrelated receive antennas and with transmit correlation. For uncorrelated transmit antennas, $\mathbf{R}_{\mathbf{t}}$ becomes an identity matrix, therefore, $\sigma_{k}^{2}=1$ in (6).

For an uncorrelated flat Rayleigh fading channel with an MU-MIMO SDM scheme and $K$ active users, the CDF of post scheduling SINR for each sub-stream is

$$
F_{\Gamma_{k}}^{M s}(\gamma)=\left(\int_{0}^{\gamma} \frac{n_{t} e^{-n_{t} \alpha / \gamma_{0}}}{\gamma_{0}\left(n_{r}-n_{t}\right) !}\left(\frac{n_{t} \alpha}{\gamma_{0}}\right)^{\left(n_{r}-n_{t}\right)} d \alpha\right)^{K} .
$$

In the case of $n_{r}=n_{t}$, the above equation can be written in a closed form as

$$
F_{\Gamma_{k}}^{M s}(\gamma)=\left(1-e^{-\frac{n_{t} \gamma}{\gamma_{0}}}\right)^{K}
$$

The PDF for the post scheduling sub-stream SINR can be derived as

$$
f_{\Gamma_{k}}^{M s}(\gamma)=\frac{d}{d \gamma} F_{\Gamma_{k}}^{M s}(\gamma)=\frac{n_{t}}{\gamma_{0}} e^{-\frac{n_{t} \gamma}{\gamma_{0}}} K\left(1-e^{-\frac{n_{t} \gamma}{\gamma_{0}}}\right)^{(K-1)} .
$$

For a dual stream MU-MIMO scheme, combining the two sub-stream SINRs of each PRB into an unified SINR with the same total (Shannon) capacity, the CDF for the post scheduling effective SINR per PRB can then be expressed as

$$
\begin{aligned}
F_{\Gamma_{u}}^{O M}(\gamma) & =\operatorname{Pr}\left(\left(\Gamma_{1}+1\right)\left(\Gamma_{2}+1\right) \leq \gamma\right) \\
& =\int_{0}^{\infty} \operatorname{Pr}\left(\Gamma_{2} \leq \frac{\gamma-x}{x+1} \mid \Gamma_{1}=x\right) f_{\Gamma_{1}}^{M s}(x) d x
\end{aligned}
$$

Under the assumption of independence between the instantaneous SINR of sub-streams, we have

$$
\begin{aligned}
F_{\Gamma_{u}}^{O M}(\gamma)= & \int_{0}^{\gamma} \frac{n_{t}}{\gamma_{0}} e^{-\frac{n_{t} x}{\gamma_{0}}} K\left(1-e^{-\frac{n_{t} x}{\gamma_{0}}}\right)^{(K-1)} \\
& \cdot\left(1-e^{-\frac{n_{t} \frac{\gamma-x}{x+1}}{\gamma_{0}}}\right)^{K} d x .
\end{aligned}
$$

\section{SINR DISTRIBUTION FOR LINEARLY PRECODED SDM MIMO SCHEMES}

With a linearly precoded MIMO scheme using the linear MMSE receiver, the received SINR at the $j$ th spatial substream of the $i$ th MS, can be related to its Mean Square Error (MSE) as [8],

$$
\Gamma_{j}=M S E_{j}^{-1}-1, \quad j \in\left\{1,2, \cdots, n_{t}\right\} .
$$


For simplicity, we have omitted the index for the $i$ th user in the above equation. For Schur-Concave objective functions, the instantaneous SINR can be obtained by minimizing the weighted sum of the MSEs as follows [8]

$$
\Gamma_{j}=\lambda_{j} \rho_{j}, \quad j \in\left\{1,2, \cdots, n_{t}\right\}
$$

where $\lambda_{j}$ is the $j$ th non-zero largest eigenvalue of the matrix $\mathbf{H}_{i} \mathbf{H}_{i}^{H} . \rho_{j}=p_{j} / N_{0}$, where $p_{j}$ is the power allocated to the $j$ th established sub-stream of the $i$ th MS and $N_{0}$ is the noise variance. It is well known that for Rayleigh MIMO fading channels, the complex matrix $\mathbf{H}_{i} \mathbf{H}_{i}^{H}$ is a complex central Wishart matrix [10]. The joint density function of the ordered eigenvalues of $\mathbf{H}_{i} \mathbf{H}_{i}^{H}$ can be expressed as [10]

$$
f_{\Lambda}=\prod_{i=1}^{M} \frac{\lambda_{i}^{N-M}}{(M-i) !(N-i) !} \prod_{i<j}^{M-1}\left(\lambda_{i}-\lambda_{j}\right)^{2} \cdot \exp \left(-\sum_{i=1}^{M} \lambda_{i}\right),
$$

where $\lambda_{1} \geq \lambda_{2} \geq \cdots \geq \lambda_{M}$. For unordered eigenvalues, the joint density function can be obtained by $f_{\boldsymbol{\Lambda}}\left(\lambda_{1}, \cdots, \lambda_{M}\right) / M$ !.

\section{A. Linearly Precoded SDM SU-MIMO Schemes}

For localized downlink transmission with linearly precoded SU-MIMO system with 2 antennas at both the transmitter and the receiver side, applying the FD PF scheduling algorithm under the assumption of identical distribution of all effective SINR for all users, the probability that the SINR of a scheduled user is below a certain threshold, i.e., the CDF of the post scheduling SINR per PRB is given by

$$
F_{\Gamma_{u}}^{C S}(\gamma)=\left[\int_{0}^{\gamma} d v \frac{1}{\left(\rho_{1} \rho_{2}\right)^{3}} \exp \left(-\frac{v}{\rho_{1}}\right) \varphi(\gamma, v)\right]^{K}
$$

where $K$ is the number of active users in the cell and

$$
\begin{aligned}
\varphi(\gamma, v) & =\rho_{2}{ }^{3} v^{2}\left[1-\exp \left(-\frac{\gamma-v}{\rho_{2}(v+1)}\right)\right]-2 \rho_{1} \rho_{2}{ }^{3} v \\
& \cdot\left[1-\exp \left(-\frac{\gamma-v}{\rho_{2}(v+1)}\right)\left(1+\frac{\gamma-v}{\rho_{2}(v+1)}\right)\right] \\
& +2 \rho_{1}{ }^{2} \rho_{2}{ }^{3}-\rho_{1}{ }^{2} \rho_{2}{ }^{3} \exp \left(-\frac{\gamma-v}{\rho_{2}(v+1)}\right) \\
& \cdot\left(\left(\frac{\gamma-v}{\rho_{2}(v+1)}\right)^{2}+\frac{2(\gamma-v)}{\rho_{2}(v+1)}+2\right)
\end{aligned}
$$

By differentiating the distribution function expressed by (15), the PDF of the effective post scheduling SINR for the linearly precoded SDM SU-MIMO scheme can be derived as

$$
\begin{aligned}
& f_{\Gamma_{u}}^{C S}(\gamma)=K \int_{0}^{\gamma} \frac{1}{\left(\rho_{1} \rho_{2}\right)^{3}(1+v)} \exp \left(-\frac{v}{\rho_{1}}-\frac{\gamma-v}{\rho_{2}(1+v)}\right) \\
& \cdot\left(\rho_{2} v-\frac{\gamma-v}{1+v} \rho_{1}\right)^{2} d v\left(\int_{0}^{\gamma} \frac{1}{\left(\rho_{1} \rho_{2}\right)^{3}} \exp \left(-\frac{v}{\rho_{1}}\right) \varphi(\gamma, v) d v\right)^{K-1}
\end{aligned}
$$

\section{B. Linearly Precoded SDM MU-MIMO Schemes}

For MU-MIMO, the distribution of instantaneous SINR for each sub-stream of each scheduled user should be computed first in order to get the distribution of the unified effective SINR for the scheduled users per PRB. This requires the derivation of the marginal PDF of each eigenvalue. The marginal density function of the $k$ th ordered eigenvalue can be obtained by

$$
\begin{aligned}
f_{\Lambda_{k}}\left(\lambda_{k}\right)= & \int_{\lambda_{k}}^{\infty} d \lambda_{k-1} \cdots \int_{\lambda_{2}}^{\infty} d \lambda_{1} \int_{0}^{\lambda_{k}} d \lambda_{k+1} \\
& \cdots \int_{0}^{\lambda_{M-1}} d \lambda_{M} f_{\boldsymbol{\Lambda}}
\end{aligned}
$$

where $f_{\Lambda}$ is given by (14). In [11], the marginal PDF of eigenvalues is approximated as

$$
f_{\Lambda_{i}}\left(\lambda_{i}\right) \simeq \frac{1}{[\beta(i)-1] !} \frac{\lambda_{i}^{\beta(i)-1}}{\tilde{\lambda}_{i}^{\beta(i)}} \exp \left(-\lambda_{i} / \tilde{\lambda}_{i}\right)
$$

where $\beta(i)=\left(n_{t}-i+1\right)\left(n_{r}-i+1\right)$ and

$$
\tilde{\lambda}_{i}=\frac{1}{\beta(i)} \bar{\lambda}_{i}=\frac{1}{\beta(i)} \int_{0}^{\infty} \lambda_{i} f_{\Lambda}\left(\lambda_{i}\right) d \lambda_{i} .
$$

Based on (13) and (19), the density function of the instantaneous SINR of the $i$ th sub-stream can be expressed as

$$
\begin{aligned}
f_{\Gamma_{i}}(\gamma) & =\frac{1}{\rho_{i}} f_{\Lambda_{i}}\left(\gamma / \rho_{i}\right) \\
& \simeq \frac{1}{\rho_{i}} \frac{1}{[\beta(i)-1] !} \frac{\left(\gamma / \rho_{i}\right)^{\beta(i)-1}}{\tilde{\lambda}_{i}^{\beta(i)}} \exp \left(-\gamma /\left(\rho_{i} \tilde{\lambda}_{i}\right)\right) .
\end{aligned}
$$

The outage probability, which is defined as the probability of the SINR being less than a targeted SINR within a specified time period, is a statistical measure of the system. From the definition, the outage probability is simply the CDF of the SINR evaluated at a targeted SINR, and can be obtained by

$$
\begin{aligned}
\operatorname{Pr}\left(\Gamma_{i} \leq \gamma\right) & =\int_{-\infty}^{\gamma} f_{\Gamma_{i}}(\alpha) d \alpha=\operatorname{Pr}\left(\lambda_{i} \leq \gamma / \rho_{i}\right) \\
& \simeq 1-\sum_{j=0}^{\beta(i)-1} \frac{\left(\gamma /\left(\rho_{i} \tilde{\lambda}_{i}\right)\right)^{j}}{j !} \exp \left(-\gamma /\left(\rho_{i} \tilde{\lambda}_{i}\right)\right) .
\end{aligned}
$$

In the multiusers case, with the MU-MIMO SDM and the FD PF packet scheduling scheme, the distribution function of the instantaneous SINR for the $i$ th sub-stream of each PRB can be obtained as

$$
F_{\Gamma_{i}}^{C M}(\gamma)=\operatorname{Pr}\left(\Gamma_{1} \leq \gamma, \cdots, \Gamma_{K} \leq \gamma\right)=\prod_{k=1}^{K} \operatorname{Pr}\left(\Gamma_{i} \leq \gamma\right)
$$

Substituting (22) into (23) yields

$$
F_{\Gamma_{i}}^{C M}(\gamma) \simeq\left[1-\sum_{j=0}^{\beta(i)-1} \frac{\left(\gamma /\left(\rho_{i} \tilde{\lambda}_{i}\right)\right)^{j}}{j !} \exp \left(-\gamma /\left(\rho_{i} \tilde{\lambda}_{i}\right)\right)\right]^{K} .
$$

Using the $K$ th order statistics [12], under the assumption of identical distribution of all effective SINR for all users, the probability density function of the instantaneous SINR of the $i$ th sub-stream of each PRB with linearly precoded MU-MIMO scheme using FD PF packet scheduling algorithm can then be obtained as

$$
\begin{aligned}
f_{\Gamma_{i}}^{C M}(\gamma)= & \frac{d}{d \gamma} F_{\Gamma_{i}(\gamma)}^{C M} \simeq \frac{K\left(\gamma / \rho_{i}\right)^{\beta(i)-1}}{\rho_{i}[\beta(i)-1] ! \tilde{\lambda}_{i}^{\beta(i)}} \exp \left(-\gamma /\left(\rho_{i} \tilde{\lambda}_{i}\right)\right) \\
\cdot & {\left[1-\sum_{j=0}^{\beta(i)-1} \frac{\left(\gamma /\left(\rho_{i} \tilde{\lambda}_{i}\right)\right)^{j}}{j !} \exp \left(-\gamma /\left(\rho_{i} \tilde{\lambda}_{i}\right)\right)\right]^{K-1} }
\end{aligned}
$$

Note that for a dual sub-stream linearly precoded SDM MUMIMO scheme with a FD PF packet scheduling algorithm, the distribution of instantaneous SINRs for the two sub-streams within a PRB are independent. We consider the baseline antenna configuration of LTE, i.e., 2 antennas at both the transmitter and the receiver side. In this case, the distribution 
function of the unified effective instantaneous SINR of the two sub-streams of each PRB can be obtained by using (10). Differentiating (10) with respect to $\gamma$ gives

$$
f_{\Gamma_{u}}^{C M}(\gamma)=\int_{0}^{\infty} \frac{1}{(x+1)} f_{\Gamma_{1}}^{C M}(x) f_{\Gamma_{2}}^{C M}\left(\frac{\gamma-x}{x+1}\right) d x
$$

Substituting (24) and (25) into (10) and limiting the integral region, we obtain

$$
\begin{aligned}
& F_{\Gamma_{u}}^{C M}(\gamma) \simeq \int_{0}^{\gamma} d x \frac{K}{\rho_{1} \tilde{\lambda}_{1}} \frac{\left(x /\left(\rho_{1} \tilde{\lambda}_{1}\right)\right)^{(\beta(i)-1)}}{(\beta(i)-1) !} e^{\left(-\frac{x}{\left(\rho_{1} \tilde{\lambda}_{1}\right)}\right)} \\
& \cdot\left[1-\sum_{j=0}^{\beta(i)-1} \frac{\left(x /\left(\rho_{1} \tilde{\lambda}_{1}\right)\right)^{j}}{j !} e^{\left(-x /\left(\rho_{1} \tilde{\lambda}_{1}\right)\right)}\right]^{K-1} \\
& \cdot\left[1-\sum_{j=0}^{\beta(2)-1} \frac{\left((\gamma-x) /\left((x+1) \rho_{2} \tilde{\lambda}_{2}\right)\right)^{j}}{j !} e^{\left(-\frac{\gamma-x}{(x+1) \rho_{2} \tilde{\lambda}_{2}}\right)}\right]^{K} .
\end{aligned}
$$

\section{Analytical Results and Conclusions}

We consider the case with 2 antennas at both the transmitter and the receiver side. Fig. 1 shows a single stream SINR and the effective SINR distribution per PRB for MIMO schemes with and without FDPS. The number of active users in the cell, which are available for scheduling is 20 . It can be seen that without packet scheduling, MU-MIMO can exploit available multiuser diversity gain, therefore it has better stream SINR distribution than SU-MIMO. For SDM SU-MIMO at 50\% percentile of effective SINR, approximately $10 \mathrm{~dB}$ gain can be obtained by using FDPS. More gain can be achieved by using MU-MIMO scheme with packet scheduling. This is due to the fact that the multiuser diversity is further exploited in SDM MU-MIMO schemes.

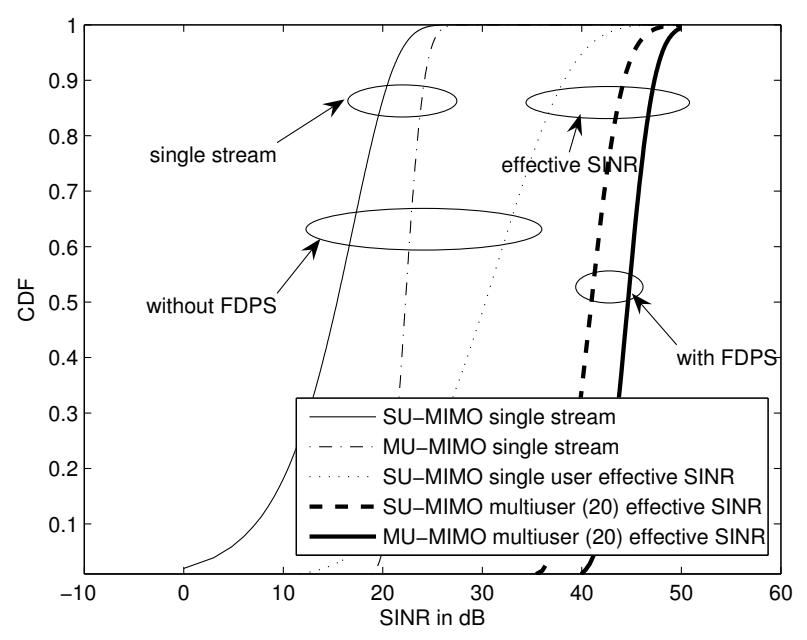

Fig. 1. SINR distribution for SDM multiuser SU and MU-MIMO schemes with 20 active users in the cell.

Fig. 2 shows the effective SINR distribution per PRB for linearly precoded SDM MIMO scheme with and without packet scheduling. The number of active users, i.e., the user diversity order, is 10 . These plots are obtained under the assumption of evenly allocated transmit power at the two transmitter antennas, and a transmitted Signal to Noise Ratio (SNR), which is defined as the total transmitted power of

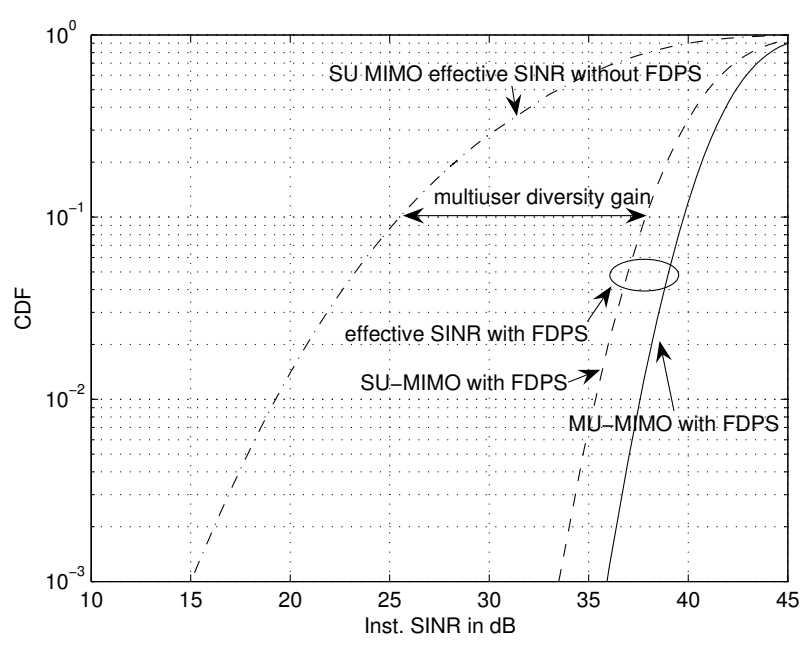

Fig. 2. SINR distribution for SDM-FDPS linearly precoded multiuser SU and MU-MIMO schemes with 10 active users in the cell.

the two sub-streams divided by the variance of the complex Gaussian noise, is equal to $20 \mathrm{~dB}$. It can be seen that for SUMIMO scheme, the multiuser diversity gain (as shown in Fig. 2) at the 10th percentile of the post scheduled SINR per PRB is about $11 \mathrm{~dB}$ with 10 users. While an MU-MIMO scheme with SDM-FDPS can achieve an additional $2 \mathrm{~dB}$ gain compared with a SDM-FDPS SU-MIMO scheme. This implies that the MU-MIMO scheme has more freedom or selection diversity than the SU-MIMO in the spatial domain.

\section{REFERENCES}

[1] 3GPP TR 25.814 V7.0.0, "Physical Layer Aspects for Evolved UTRA," Tech. Rep., June 2006.

[2] N. Wei, A. Pokhariyal, T. B. Sørensen, T. E. Kolding, and P. E. Mogensen, "Performance of MIMO with Frequency Domain Packet Scheduling in UTRAN LTE downlink," in IEEE VTC, Apr. 2007, pp. $1177-1181$.

[3] T. Park, O.-S. Shin, and K. B. Lee, "Proportional Fair Scheduling for Wireless Communication with Multiple Transmit and Receive Antennas," in IEEE VTC, Florida, USA, October 2003, vol. 3, pp. 1573 - 1577.

[4] V. K. N. Lau, "Proportional fair space-time scheudling for wireless communications," IEEE Trans. Commun., vol. 53, no. 8, pp. 1353-1360, Aug. 2005.

[5] J. M. Holtzman, "CDMA forward link waterfilling power control," in IEEE Vehicular Technology Conference (VTC), May 2000, pp. 16361667, Toyko, Japan.

[6] N. Wei, T. B. Sørensen, T. E. Kolding, and P. E. Mogensen, "Analysis and Evalution of Link Adaptation with MIMO adaptation," in IEEE VTC, Sept. 2006, pp. 1-5.

[7] 3GPP TR 25.214 V4.4.0, "Physical Layer Procedures (FDD)," Tech. Rep., Mar. 2002.

[8] D. P. Palomar, J. M. Cioffi, and M. A. Lagunas, "Joint Tx-Rx Beamforming Design for Multicarrier MIMO Channels: A Unified Framework for Convex Optimization," IEEE Transaction on Signal Processing, vol. 51, no. 9, pp. 2381-2401, Sept. 2003.

[9] D. A. Gore, Jr. R. W. Heath, and A. J. Paulraj, "Transmit Selection in Spatial Multiplexing Systems," IEEE Communications Letters, vol. 6, no. 11, pp. 491-493, Nov. 2002.

[10] E. Edelman, "Eigenvalues and condition numbers of random matrices," SLAM J. Matrix Anal. Appl., vol. 9, no. 4, pp. 543-559, Oct. 1988.

[11] T. Taniguchi, S. Sha, and Y. Karasawa, "Statistical Distribution of Eigenvalues of Correlation Matrices in i.i.d MIMO Channels under Rayleigh Fading," in IEEE International Symposium on Personal, Indoor and Mobile Radio Communications, 2005, pp. 537-541.

[12] H. A. David, Order Statistics, Wiley, 1980. 